\title{
Une infinité de structures de contact tendues sur les variétés toroïdales
}

Vincent Colin

\begin{abstract}
We show that every closed, toroidal, irreducible, orientable 3 -manifold carries infinitely many universally tight contact structures.

Résumé. On démontre que toute variété orientable, irréductible, close et toroïdale de dimension 3 porte une infinité de structures de contact universellement tendues.
\end{abstract}

Mathematics Subject Classification (2000). 57R17, 53D10.

Mots-clés. Variété de contact, structure tendue, torsion, variété toroïdale.

\section{Introduction}

Une variété de dimension 3 est dite torö̈dale si elle contient un tore incompressible, c'est-à-dire plongé et $\pi_{1}$-injecté. L'objectif de ce texte est de démontrer le théorème suivant, qui répond partiellement à une conjecture d'E. Giroux [Gi5, Gi6] pronostiquant qu 'une variété de dimension 3-orientable, irréductible et close porte une infinité de structures de contact tendues si et seulement si elle est toroïdale :

Théorème 1.1. Toute variété de dimension 3 , orientable, irréductible, close (compacte sans bord) et toroïdale porte une infinité de structures de contact universellement tendues deux à deux non isomorphes.

Jusqu'ici, les principaux résultats établis concernaient les fibrés en tores sur le cercle [Gi3, Gi4, Gi5] et les variétés irréductibles contenant un tore normal [Co2]: un tore est normal s'il est incompressible et si on ne peut pas le disjoindre par isotopie d'un autre tore incompressible. Cette propriété caractérise des variétés dans lesquelles se plongent de manière $\pi_{1}$-injective un fibré de Seifert de base "assez large". Dans [Co2], on démontre ainsi que toute variété orientable et irréductible qui contient un tore normal porte une infinité de structures de contact universellement tendues.

Comme dans ce premier travail, la démonstration du théorème 1.1 repose sur la notion de torsion, introduite par E. Giroux [Gi3, Gi4, Gi5], et sur l'étude qu'il 
en a faite dans le cas du tore épais.

Avant de poursuivre plus avant la discussion, on rappelle quelques notions de géométrie de contact.

Une structure de contact sur une variété $\mathrm{V}$ de dimension 3 est un champ de plans $\xi$ qui possède une équation locale $\alpha=0$ telle que la 3 -forme $\alpha \wedge d \alpha$ soit une forme de volume. En particulier, sur une variété orientée, toute structure de contact possède un signe (donné par celui de $\alpha \wedge d \alpha$ qui se révèle indépendant du choix de $\alpha$ ). Par défaut, toutes les variétés de contact rencontrées par la suite sont supposées orientées et les structures de contact positives.

En dimension 3, les structures de contact se scindent en deux catégories complémentaires: les structures vrillées et les structures tendues. Une structure $\xi$ sur une variété $\mathrm{V}$ est vrillée s'il existe un disque $\mathrm{D}$ plongé dans $\mathrm{V}$ tel que $\left.\xi\right|_{\partial \mathrm{D}}=\mathrm{T}_{\partial \mathrm{D}} \mathrm{D}$. Si le rappel dans tout revêtement de $\mathrm{V}$ d'une structure vrillée est une structure vrillée, ce n'est pas toujours le cas des structures tendues (voir par exemple [Co1]) ; c'est pourquoi on distingue comme sous-catégorie de ces dernières les structures universellement tendues, qui restent tendues après un passage au revêtement universel. Aujourd'hui, les structures vrillées sont parfaitement comprises grâce au travail de Y. Eliashberg [El1]. Les structures tendues en revanche restent en partie mystérieuses. Leur étude est d'autant plus intéressante qu'elle se situe à l'intersection de nombreuses branches de la géométrie: théorie des nœuds, théorie des feuilletages, géométrie symplectique...

On décrit à présent des outils intervenant dans l'étude des variétés de contact.

Si $\mathrm{S}$ est une surface orientée dans $\mathrm{V}$ et si $\xi$ est orientée près de $\mathrm{S}$, en dehors des points $\left(x_{i}\right)_{i \in \mathrm{I}}$ de $\mathrm{S}$, génériquement isolés, où $\xi\left(x_{i}\right)=\mathrm{T}_{x_{i}} \mathrm{~S}$, l'intersection de $\xi$ et de TS est un champ de droites orientées qui s'intègre en un feuilletage de $\mathrm{S}$, appelé feuilletage caractéristique de $\mathrm{S}$ et noté $\xi \mathrm{S}$. Il est singulier en les points $x_{i}$. Le feuilletage caractéristique tracé par une structure $\xi$ sur une surface $\mathrm{S}$ détermine $\xi$ près de $\mathrm{S}$. En particulier, pour recoller deux structures de contact de même signe situées de part et d'autre d'une surface, il suffit que leurs feuilletages caractéristiques coïncident.

Toute courbe intégrale de $\xi$ est dite legendrienne. Si une courbe legendrienne orientée $\gamma$ est contenue dans une surface $\mathrm{S}$, on définit son invariant de ThurstonBennequin relatif, noté $t b(\gamma, \mathrm{S})$, comme la moitié de la somme algébrique, le long de $\gamma$, des intersections entre $\xi$ et TS. Si $\gamma$ est une courbe positivement transverse à $\xi$ et borde une surface orientée $\mathrm{S}$, le champ de plans $\left.\xi\right|_{\mathrm{S}}$ admet une section non singulière $X$. On appelle autoenlacement de $\gamma$, noté $l(\gamma)$, l'enlacement entre $\gamma$ et toute courbe obtenue en poussant un peu $\gamma$ par $X$.

Pour différencier les structures de contact tendues, E. Giroux [Gi3, Gi4, Gi5] a introduit la notion de torsion. Si $\mathrm{T} \subset(\mathrm{V}, \xi)$ est un tore incompressible dans une variété de contact, on cherche a plonger, pour le plus grand entier naturel $n$ possible, le produit de contact $\left(\mathrm{T}^{2} \times[0,2 \pi], \operatorname{ker}(\cos n t d x+\sin n t d y)\right)$ dans la même classe d'isotopie $\mathrm{C}$ que celle d'un voisinage tubulaire de $\mathrm{T}$. Cet entier (éventuellement nul, si de tels plongements n'existent pas, ou infini) est la torsion 
de $\xi$ dans la classe $\mathrm{C}$ et est noté $\operatorname{Tor}(\mathrm{V}, \xi, \mathrm{C})$, ou $\operatorname{Tor}(\mathrm{V}, \xi)$ s'il n'y a pas d'ambiguïté. On a vu dans [Co2] comment lire cet invariant sur le nombre de Thurston-Bennequin relatif d'un certain type de courbe. C'est cette stratégie que l'on va à nouveau appliquer pour démontrer le théorème 1.1.

Pour de plus amples détails sur les notions de base de la géométrie de contact, et notamment sur la création et l'élimination de singularités d'un feuilletage $\xi \mathrm{S}$ par isotopie de $\mathrm{S}$, on renvoie à [Gi2].

Le théorème 1.1 a été tout récemment annoncé par K. Honda, W. Kazez et G. Matić.

Je remercie chaleureusement Emmanuel Giroux de l'aide qu'il m'a apportée dans la recherche de cette démonstration. Je lui dois notamment l'idée de se placer sur un revêtement de groupe $\pi_{1}(\mathrm{~T})$ ainsi que de distinguer les différentes possibilités pour le rappel de $\mathrm{T}$. Une partie de la rédaction de ce texte a été effectuée lors d'un séjour à l'université de Stanford et à l'American Institute of Mathematics. Je remercie ces deux institutions de leur soutien.

\section{Recueil de résultats sur la torsion}

L'objet de cette section est de répertorier des résultats, principalement dus à E. Giroux, K. Honda et Y. Eliashberg, concernant la torsion des structures de contact universellement tendues sur le tore épais, l'anneau épaissi et le demiespace. On donne si nécessaire des esquisses de preuves permettant d'expliciter le lien à ces travaux lorsqu'il n'est pas totalement transparent. Le lemme 2.8 peut être également déduit des arguments développés dans [Co2].

\subsection{Structures de contact universellement tendues sur le tore épais}

\section{A. Calcul de la torsion sur un modèle}

Lemme 2.1. [Gi5] Soit $\xi$ la structure de contact définie sur $\mathrm{T}^{2} \times[-1,1]=$ $\{(x, y, \theta)\}$ par l'équation $\cos f(\theta) d x+\sin f(\theta) d y=0$, où $f:[-1,1] \rightarrow \mathbf{R}$ est ̀̀ dérivée strictement positive et vérifie: $f(-1) \in[0,2 \pi[$ et $f(1) \in[2 n \pi, 2(n+1) \pi[$, $n \in \mathbf{N}$. Alors $\operatorname{Tor}\left(\mathrm{T}^{2} \times[-1,1], \xi\right)$ est la partie entière de $(f(1)-f(-1)) / 2 \pi$, c'est-à-dire $n$ ou $n-1$.

\section{B. Addition des torsions}

Lemme 2.2. [Gi5] Soit $\xi$ une structure de contact universellement tendue sur $\mathrm{T}^{2} \times \mathbf{R}$ telle que $\xi \mathrm{T}^{2} \times\{ \pm 1\}$ soit un feuilletage linéaire. On a:

$$
\begin{gathered}
\left.\left.\operatorname{Tor}\left(\mathrm{T}^{2} \times \mathbf{R},\left.\xi\right|_{\mathrm{T}^{2} \times \mathbf{R}}\right)=\operatorname{Tor}\left(\mathrm{T}^{2} \times\right]-\infty,-1\right],\left.\xi\right|_{\left.\left.\mathrm{T}^{2} \times\right]-\infty,-1\right]}\right) \\
+\operatorname{Tor}\left(\mathrm{T}^{2} \times[-1,1],\left.\xi\right|_{\mathrm{T}^{2} \times[-1,1]}\right)+\operatorname{Tor}\left(\mathrm{T}^{2} \times\left[1,+\infty\left[,\left.\xi\right|_{\mathrm{T}^{2} \times[1,+\infty[}\right)+k,\right.\right.
\end{gathered}
$$

où $k=0,1$, ou 2 . 


\section{Critère d'annulation de la torsion}

Lemme 2.3. Soit $\xi$ une structure universellement tendue sur $\mathrm{T}^{2} \times[-1,1]$ qui trace sur $\mathrm{T}^{2} \times\{1\}$ un feuilletage sans singularité ni composante de Reeb avec un nombre fini d'orbites périodiques non dégénérées et un feuilletage linéaire sur $\mathrm{T}^{2} \times\{-1\}$. On note $\mathrm{A}=\mathrm{S}^{1} \times\{*\} \times[-1,1] \subset \mathrm{T}^{2} \times[-1,1]$. On suppose que $\partial \mathrm{A}$ est transverse, ou éventuellement tangent le long de $\mathrm{T}^{2} \times\{-1\} \cap \partial \mathrm{A}, \grave{a} \xi$. S'il existe un arc legendrien $\gamma$ plongé dans $\mathrm{A}$ joignant un bord de $\mathrm{A}$ à l'autre et tel que $t b(\gamma, \mathrm{A})=-\frac{1}{2}$, alors $\operatorname{Tor}\left(\mathrm{T}^{2} \times[-1,1], \xi\right)=0$.

Démonstration. On peut toujours supposer que, quitte à changer A en conservant $\gamma$, le bord de A est parallèle aux orbites de $\xi \mathrm{T}^{2} \times\{1\}$. Après cette modification, on est assuré que $t b(\gamma, \mathrm{A})=0,-\frac{1}{2}$ ou -1 . Dès lors, pour tout feuilletage non singulier $\mathrm{F}$ de $\mathrm{T}^{2} \times\{1\}$, transverse à $\partial \mathrm{A}$, dont les orbites fermées sont non dégénérées et coïncident avec celles de A (avec une orientation éventuellement différente, mais les orbites attractives restent attractives et les répulsives, répulsives), il existe (voir [Gi1]) une isotopie $\mathrm{C}^{0}$-petite de $\mathrm{T}^{2} \times\{1\}$ fixant $\mathrm{A}$, telle que le feuilletage caractéristique du nouveau tore soit $\mathrm{F}$ (ce feuilletage comporte éventuellement des composantes de Reeb). Ce faisant, d'après [Gi5], la torsion du produit délimité par $\mathrm{T}^{2} \times\{-1\}$ et l'image de $\mathrm{T}^{2} \times\{1\}$ est la même que celle de $\left(\mathrm{T}^{2} \times[-1,1], \xi\right)$. On note $\left(\mathrm{T}^{2} \times[-1,1], \xi_{\mathrm{F}}\right)$ ce nouveau produit. Par construction, l'anneau A et l'arc legendrien $\gamma$ inclus dans A joignent tous deux un bord de $\left(\mathrm{T}^{2} \times[-1,1], \xi_{\mathrm{F}}\right)$ à l'autre. On a de plus $t b(\gamma, \mathrm{A})=0,-\frac{1}{2}$ ou -1 .

Toujours d'après [Gi5] et sa description complète des structures de contact tendues sur le tore épais, si $n$ désigne la torsion de $\xi$, il existe un feuilletage $\mathrm{F}$ de $\mathrm{T}^{2} \times\{1\}$, un entier $k$, un réel $\theta_{0}$ et une fonction $f$ strictement supérieure à $2 n \pi$ tels que $\left(\mathrm{T}^{2} \times[-1,1], \xi_{\mathrm{F}}\right) \simeq\{(x, y, \theta \leq f(y))\} \subset\left(\mathrm{T}^{2} \times[0,2(n+k) \pi], \operatorname{ker}(\cos (\theta+\right.$ $\left.\left.\left.\theta_{0}\right) d x+\sin \left(\theta+\theta_{0}\right) d y\right)\right)$. Dans ces coordonnées, on prolonge A par l'anneau produit $(\partial \mathrm{A} \cap\{\theta=f(y)\}) \times\{f(y) \leq \theta \leq 2(n+k) \pi\}$, et l'arc $\gamma_{\mathrm{F}} \operatorname{par}\left(\partial \gamma_{\mathrm{F}} \cap\{\theta=\right.$ $f(y)\}) \times\{f(y) \leq \theta \leq 2(n+k) \pi\}$. On obtient ainsi un anneau $\mathrm{A}_{\mathrm{F}}$ portant un arc legendrien $\gamma_{\mathrm{F}}$ qui joignent tous deux un bord à l'autre de $\mathrm{T}^{2} \times[0,2(n+k) \pi]$. Par construction, $t b\left(\gamma_{\mathrm{F}}, \mathrm{A}_{\mathrm{F}}\right) \geq-k-\frac{1}{2}$ et, comme $\partial \mathrm{A}_{\mathrm{F}}$ est transverse à $\xi$, on peut également supposer, quitte à effectuer une isotopie de $A_{F}$ relative à $\gamma_{F}$ ne modifiant pas $t b\left(\gamma_{\mathrm{F}}, \mathrm{A}_{\mathrm{F}}\right)$, que $\partial \mathrm{A}_{\mathrm{F}}$ est transverse (ou éventuellement partout tangent) à la structure.

D'après [Ka] (théorème 7.6), on a alors $t b\left(\gamma_{\mathrm{F}}, \mathrm{A}_{\mathrm{F}}\right) \leq-n-k$. On en déduit que $n \leq \frac{1}{2}$, ce qui implique que l'entier naturel $n$ est nul.

\section{Lemme de réalisation}

Lemme 2.4. [Gi5] Soit $\xi$ une structure de contact universellement tendue sur $\mathrm{T}^{2} \times\left[0,+\infty\left[\right.\right.$ qui trace un feuilletage linéaire sur $\mathrm{T}^{2} \times\{0\}$. Si $\xi$ est de torsion finie $n$, il existe $\left(c, \theta_{0}\right) \in \mathbf{R}^{2}$ tel que pour tout $k \in \mathbf{N}$, il existe un plongement $\phi$ de $\mathrm{T}^{2} \times[0, k]$ dans $\mathrm{T}^{2} \times[0, c]$ laissant invariant $\mathrm{T}^{2} \times\{0\}$, et tel que $\phi_{*} \xi$ 
ait pour équation $\cos \left(\theta+\theta_{0}\right) d x+\sin \left(\theta+\theta_{0}\right) d y=0$. On peut même imposer que $c=2 \pi(n+2)$.

On indique simplement comment se ramener à des résultats d'E. Giroux.

D'après [Gi1], quitte à effectuer des isotopies $\mathrm{C}^{0}$-petites sur les tores $\mathrm{T}^{2} \times\{k\}$, on peut toujours supposer que le feuilletage $\xi \mathrm{T}^{2} \times\{k\}$ comporte un nombre fini d'orbites périodiques non dégénérées. On se place dans cette situation. La structure $\left.\xi\right|_{\mathrm{T}^{2} \times[0, k]}$ est alors complètement décrite (voir [Gi5]) par — outre la torsion $n$ et $\xi \mathrm{T}^{2} \times\{i\}$, pour $i=0, k$ - la donnée d'une famille d'anneaux $\left(\mathrm{A}_{i}\right)_{1 \leq i \leq k}$ deux à deux disjoints dans $\mathrm{T}^{2} \times[0, k]$, s'appuyant sur les orbites périodiques de $\xi \mathrm{T}^{2} \times\{k\}$. On considère le produit $\mathrm{T}^{2} \times[0, k]$ comme naturellement inclus dans $\mathrm{T}^{2} \times[0, k+1]$. Toujours d'après [Gi5], plonger $\left(\mathrm{T}^{2} \times[0, k], \xi\right)$ dans $\left(\mathrm{T}^{2} \times[0,2 \pi(n+2)], \operatorname{ker}\left(\cos \left(\theta+\theta_{0}\right) d x+\sin \left(\theta+\theta_{0}\right) d y\right)\right)$ revient a trouver une famille d'anneaux $\left(\mathrm{A}_{i}^{\prime}\right)_{1 \leq i \leq l}$ deux à deux disjoints dans $\mathrm{T}^{2} \times[k, k+1]$ dont les bords coïncident avec ceux des anneaux de la famille $\left(\mathrm{A}_{i}\right)_{1<i<l}$ et telle que la réunion des deux familles constitue un ou deux tores incompressibles dans $\mathrm{T}^{2} \times[0, k+1]$.

En d'autres termes, la preuve du lemme 2.4 se réduit à la démonstration du lemme suivant, laissée à la sagacité du lecteur:

Lemme 2.5. Soit $\left(\alpha_{i}\right)_{1 \leq i \leq l}$ une famille d'arcs deux à deux disjoints inclus dans $\mathrm{S}^{1} \times[0,1]$, dont les extrémités sont incluses dans $\mathrm{S}^{1} \times\{1\}$. Il existe une famille d'arcs $\left(\alpha_{i}^{\prime}\right)_{1 \leq i \leq l}$ inclus dans $\mathrm{S}^{1} \times[1,2]$, dont les extrémités coïncident avec celles des arcs de la famille $\left(\alpha_{i}\right)_{1 \leq i \leq l}$, et telle que la réunion des familles $\left(\alpha_{i}\right)_{1 \leq i \leq l}$ et $\left(\alpha_{i}^{\prime}\right)_{1 \leq i \leq l}$ forme un ou deux cercles non contractiles dans $\mathrm{S}^{1} \times[0,2]$.

\section{E. Élimination des singularités}

Lemme 2.6. Soient $\xi$ une structure universellement tendue sur $\mathrm{V}=\mathrm{T}^{2} \times \mathbf{R}$, et $\mathrm{A}=\mathrm{S}^{1} \times\{*\} \times[-1,1] \subset \mathrm{T}^{2} \times[-1,1] \subset \mathrm{T}^{2} \times \mathbf{R}$. On suppose que $\partial \mathrm{A}$ est transverse $\grave{a} \xi \mathrm{A}$ avec un signe constant et que $\xi \mathrm{A}$ ne contient que des singularités de même signe. On supppose de plus que $\xi \mathrm{T}^{2} \times\{1\}$ est un feuilletage sans singularité ni composante de Reeb.

Il existe une isotopie $\mathrm{C}^{0}$-petite de $\mathrm{T}^{2} \times[-1,1]$ fixant un voisinage de $\mathrm{A} \cup$ $\mathrm{T}^{2} \times\{1\}$ telle que l'image de $\mathrm{T}^{2} \times\{-1\}$ porte un feuilletage sans singularité ni composante de Reeb.

Démonstration. On utilise des techniques développées dans [Gi1]. On se place sur un revêtement de degré deux $p: \mathrm{V}^{\prime} \rightarrow \mathrm{V}$ de $\mathrm{V}$ dans lequel la préimage de A est constituée de deux anneaux disjoints $A_{1}$ et $A_{2}$. En particulier, dans ce revêtement, $p^{-1}(\mathrm{~A})$ découpe $p^{-1}\left(\mathrm{~T}^{2} \times[-1,1]\right)$ en deux tores solides, dont un est dénoté $\mathrm{T}$. Le bord de $\mathrm{T}$ est constitué de la réunion de $p^{-1}(\mathrm{~A})$ et de deux anneau $\mathrm{B}_{-1}$ et $\mathrm{B}_{1}$ qui sont envoyés par $p$ respectivement sur $\mathrm{T}^{2} \times\{-1\}$ et $\mathrm{T}^{2} \times\{1\}$. On sait par hypothèse que, si $\xi^{\prime}$ désigne le rappel de $\xi$ dans $\mathrm{V}^{\prime}$, le feuilletage $\xi^{\prime}\left(\mathrm{A}_{2} \cup \mathrm{B}_{1}\right)$ ne porte que des singularités de même signe et est soit sortant, soit rentrant le long du bord. En particulier, $l\left(\partial\left(\mathrm{A}_{2} \cup \mathrm{B}_{1}\right)\right)=0$. On a donc aussi $l\left(\partial\left(\mathrm{A}_{1} \cup \mathrm{B}_{-1}\right)\right)=0$. Comme $\xi^{\prime} \mathrm{A}_{1}$ ne présente que des singularités de même 
signe et que $\xi^{\prime} \mathrm{A}_{1}$ est soit rentrant, soit sortant le long de $\partial \mathrm{A}_{1}$, ces singularités s'éliminent toutes par une isotopie de $A_{1}$ fixant $B_{-1}$ (voir [Gi1]). On en déduit que les singularités de $B_{-1}$ s'éliminent par une isotopie $C^{0}$-petite de $B_{-1}$ fixant un voisinage de son bord (voir [Gi1]), ce qui, transcrit dans $\mathrm{V}$, fournit le résultat recherché.

\subsection{Structures universellement tendues sur $\mathbf{R} \times \mathrm{S}^{1} \times[0,1]$}

On étend la notion de torsion aux structures définies sur, respectivement, $\mathbf{R} \times \mathrm{S}^{1} \times$ $\mathrm{S}^{1}=\{(x, y, \theta)\}$ et $\mathbf{R} \times \mathrm{S}^{1} \times[0,1]$ (avec des coordonnées similaires), en remplaçant la recherche de plongements de $\mathrm{T}^{2} \times[0,2 \pi]$ par celle de plongements (propres et $\pi_{1}$-injectifs $)$ de $\left(\mathbf{R} \times \mathrm{S}^{1} \times[0,2 \pi], \operatorname{ker}(\cos n \theta d x+\sin n \theta d y)\right)$ respectivement non séparant et parallèles au bord. On parle alors de torsion annulaire. Le résultat suivant découle de [Co2]. Il peut être également déduit des techniques développées dans [Gi5].

Lemme 2.7. [Co2, Gi5] Toute structure de contact universellement tendue sur $\mathbf{R} \times \mathrm{S}^{1} \times \mathrm{S}^{1}$ est de torsion annulaire finie.

Corollaire 2.8. Toute structure de contact universellement tendue $\xi$ sur $\mathrm{V}=$ $\mathbf{R} \times \mathrm{S}^{1} \times[-1,1]=\{(x, y, \theta)\}$ qui a pour équation $\cos f(x, y, \theta) d x+\sin f(x, y, \theta) d y=$ 0 sur $\mathbf{R} \times \mathrm{S}^{1} \times[-1,-1+\epsilon] \cup \mathbf{R} \times \mathrm{S}^{1} \times[1-\epsilon, 1]$, où $f: \mathbf{R} \times \mathrm{S}^{1} \times[-1,-1+\epsilon] \cup$ $\mathbf{R} \times \mathrm{S}^{1} \times[1-\epsilon, 1] \rightarrow[a, b]$ vérifie $f(x, y, \theta)=f(\theta)$ sur $\mathbf{R} \times \mathrm{S}^{1} \times\left[-1+\epsilon-\epsilon^{\prime},-1+\right.$ $\epsilon] \cup \mathbf{R} \times \mathrm{S}^{1} \times\left[1-\epsilon, 1-\epsilon+\epsilon^{\prime}\right]$, a une torsion annulaire finie.

Démonstration. L'image de $f$ est incluse par hypothèse dans un intervalle $[a, b]$ compact. On note $g: \mathbf{R} \times \mathrm{S}^{1} \times[2,3] \rightarrow \mathbf{R}$ une fonction dont la dérivée partielle par rapport à la troisième variable est strictement positive avec $g(x, y, 2)=$ $f(x, y, 1) \bmod 2 \pi$ et $g(x, y, 3)=f(x, y,-1) \bmod 2 \pi$. Le champ de plans $\zeta$ donné par l'équation $\cos g(x, y, \theta) d x+\sin g(x, y, \theta) d y=0$ est une structure de contact sur $\mathbf{R} \times \mathrm{S}^{1} \times[2,3]=\{(x, y, \theta)\}$. En identifiant $\mathbf{R} \times \mathrm{S}^{1} \times\{1\} \subset \mathrm{V}$ avec $\mathbf{R} \times \mathrm{S}^{1} \times\{2\}$ d'une part, et $\mathbf{R} \times \mathrm{S}^{1} \times\{-1\} \subset \mathrm{V}$ avec $\mathbf{R} \times \mathrm{S}^{1} \times\{3\} \subset \mathrm{V}$ d'autre part, on obtient, en prolongeant $\xi$ par $\zeta$, une structure de contact $\eta$ sur $\mathrm{W}=\mathbf{R} \times \mathrm{S}^{1} \times \mathrm{S}^{1}$. La structure $\eta$ est universellement tendue par application d'une version annulaire du théorème de recollement 4.3 (voir [Co1]). On applique alors le lemme 2.7 pour conclure.

\subsection{Structures tendues sur le demi-espace $\mathbf{R}^{2} \times[0,+\infty[$}

Sur le demi-espace $\mathbf{R}^{2} \times[0,+\infty[$, la notion de torsion disparaît comme le montre le théorème de classification suivant, dû à Y. Eliashberg.

Lemme 2.9. [E-T] Si $\xi$ est une structure tendue sur $\mathbf{R}^{2} \times[0,+\infty[=\{(x, y, t)\}$ qui a pour équation $\cos t d x+\sin t d y=0$ sur $\mathbf{R}^{2} \times[0, \epsilon[$, alors elle est conjuguée 
à la structure définie globalement par la même équation par un difféomorphisme qui est l'identité près du bord.

\section{Décomposition topologique du problème}

Soit V une variété de dimension 3 -orientable, irréductible et close qui contient un tore incompressible.

On sait d'après W. Jaco, P. Shalen et K. Johannson [Ja, J-S, Jo] qu'il existe une collection minimale finie $\left(\mathrm{T}_{i}\right)_{0 \leq i \leq n}$ de tores incompressibles deux à deux disjoints, unique à permutation et isotopie près, telle que toute composante de $\mathrm{V} \backslash\left(\cup_{0 \leq i \leq n} \mathrm{~T}_{i}\right)$ soit ou un fibré de Seifert, ou atoroïdale (c'est-à-dire dans laquelle tout tore incompressible est parallèle à une composante de bord).

Si V est un fibré de Seifert, on note $\mathrm{T}$ un tore incompressible quelconque de $\mathrm{V}$. Dans le cas contraire, la collection $\left(\mathrm{T}_{i}\right)_{0 \leq i \leq n}$ découpant $\mathrm{V}$ est non vide. On distingue alors deux types de variétés:

- Type A: une des composantes de $\mathrm{V} \backslash\left(\cup_{0 \leq i \leq n} \mathrm{~T}_{i}\right)$ est atoroïdale (et n'est pas un fibré de Seifert) ; on note $\mathrm{T}$ un tore de la famille $\left(\mathrm{T}_{i}\right)_{0 \leq i \leq n}$ adjacent à une de ces composantes ;

- Type B (variétés graphées): toutes les composantes de $\mathrm{V} \backslash\left(\cup_{0 \leq i \leq n} \mathrm{~T}_{i}\right)$ sont des fibrés de Seifert; on pose $\mathrm{T}=\mathrm{T}_{0}$.

On note $\pi: \overline{\mathrm{V}} \rightarrow \mathrm{V}$ le revêtement de $\mathrm{V}$ de groupe $\pi_{1}(\mathrm{~T})$.

Dans le cas A, deux situations peuvent se produire:

- a) tous les relevés de $\mathrm{T}$ dans $\overline{\mathrm{V}}$ sauf un sont des plans ;

- b) il existe un revêtement $p: \tilde{\mathrm{V}} \rightarrow \mathrm{V}$ de groupe $\mathbf{Z}$ dans lequel deux relevés $\tilde{T}_{1}$ et $\tilde{\mathrm{T}}_{2}$ de $\mathrm{T}$ sont des anneaux conjugués à $\mathrm{S}^{1} \times \mathbf{R}$.

On note $\overline{\mathrm{T}}$ un relevé compact de $\mathrm{T}$ dans $\overline{\mathrm{V}}$.

Remarque 3.1. Tout tore incompressible non isotope à l'un des tores $T_{i}$ de la décomposition de Jaco-Shalen-Johannson est automatiquement normal. Si un tel tore existe, la démonstration du théorème 1.1 découle alors de [Co2]. Les seuls cas à considérer sont donc ceux où les composantes de Seifert ont pour base soit une sphère épointée, la somme du nombre de points retirés et du nombre de fibres singulières étant inférieure à 3 , soit le plan projectif épointé, la somme du nombre de points retirés et du nombre de fibres singulières étant inférieure à 2 . Les arguments développés par la suite ne tiennent pas compte de cette réduction.

Les deux lemmes qui suivent concernent les couples $(\mathrm{V}, \mathrm{T})$ de type A.

Lemme 3.2. Dans le cas a), on note $\overline{\mathrm{V}}_{1}$ et $\overline{\mathrm{V}}_{2}$ l'adhérence dans $\overline{\mathrm{V}}$ des deux composantes de $\pi^{-1}(\mathrm{~V} \backslash \mathrm{T})$ adjacentes à $\overline{\mathrm{T}}$. La variété $\overline{\mathrm{V}}$ est alors obtenue en recollant à chaque composante de $\partial\left(\overline{\mathrm{V}}_{1} \cup \overline{\mathrm{V}}_{2}\right)$ un demi-espace $\left(\simeq \mathbf{R}^{2} \times[0, \infty[)\right.$. En particulier, $\overline{\mathrm{V}}$ est difféomorphe à l'intérieur de $\overline{\mathrm{V}}_{1} \cup \overline{\mathrm{V}}_{2}$ dans $\overline{\mathrm{V}}$. Topologiquement, elle est également conjuguée à $\mathrm{T}^{2} \times \mathbf{R}$.

Démonstration. Comme $\mathrm{V}$ est Haken, elle est revêtue par $\mathbf{R}^{3}$ (voir par exemple $[\mathrm{He}]$ ) et tout relevé $\mathrm{P}$ de $\mathrm{T}$ dans $\mathbf{R}^{3}$ découpe $\mathbf{R}^{3}$ en deux demi-espaces. En 
recollant, suivant les cas, un de ces deux demi-espaces aux composantes de bord non compactes de $\bar{V}_{1}$ et $\bar{V}_{2}$, on obtient un revêtement de $\mathrm{V}$, qui est de groupe $\pi_{1}(\mathrm{~T})$ d'après le théorème de Van Kampen. Il est donc conjugué à $\overline{\mathrm{V}}$.

Le fait que $\overline{\mathrm{V}}$ soit conjugué à $\mathrm{T}^{2} \times \mathbf{R}$ est un résultat classique de topologie démontré dans $[\mathrm{Si}, \mathrm{Ja}]$.

De la même manière, on montre le résultat suivant:

Lemme 3.3. Dans le cas b), la variété $\tilde{\mathrm{V}}$ est conjuguée à $\mathbf{R} \times \mathrm{S}^{1} \times \mathbf{R}$ et les deux anneaux $\tilde{\mathrm{T}}_{1}$ et $\tilde{\mathrm{T}}_{2}$ à $\mathbf{R} \times \mathrm{S}^{1} \times\{-1\}$ et $\mathbf{R} \times \mathrm{S}^{1} \times\{1\}$. On note $\tilde{\mathrm{V}}_{1}$ et $\tilde{\mathrm{V}}_{2}$ l'adhérence des composantes de $p^{-1}(\mathrm{~V} \backslash \mathrm{T})$ adjacentes à, respectivement, $\tilde{\mathrm{T}}_{1}$ et $\tilde{\mathrm{T}}_{2}$, et qui ne rencontrent pas le produit $\left.\mathbf{R} \times \mathrm{S}^{1} \times\right]-1,1[$. Nécessairement, une composante de $\mathrm{V} \backslash\left(\cup_{0 \leq i \leq n} \mathrm{~T}_{i}\right)$ adjacente à $\mathrm{T}$ est un fibré de Seifert. Deux cas peuvent alors se produire:

- $b_{1}$ ) La composante de Seifert adjacente à $\mathrm{T}$ ne fibre pas au-dessus d'une bande de Möebius et, pour $i=1,2$, les composantes de $\partial \tilde{V}_{i} \backslash \tilde{T}_{i}$ sont toutes des plans. La variété $\tilde{\mathrm{V}}$ est alors obtenue à partir de $\mathbf{R} \times \mathrm{S}^{1} \times[-1,1]$ en recollant $\tilde{\mathrm{V}}_{1}$ sur $\mathrm{T}^{2} \times\{-1\}$ et $\tilde{\mathrm{V}}_{2}$ sur $\mathrm{T}^{2} \times\{1\}$, puis en recollant à chaque composante de bord restante de $\tilde{\mathrm{V}}_{1}$ et $\tilde{\mathrm{V}}_{2}$ des demi-espaces $\left(\simeq \mathbf{R}^{2} \times[0, \infty[)\right.$. Dans ce cas, on note $q: \tilde{\mathrm{V}} \rightarrow \overline{\mathrm{V}}$ l'application de revêtement qui envoie $\tilde{\mathrm{T}}_{1}$ sur $\overline{\mathrm{T}}$. L'image de $\tilde{\mathrm{V}}_{1}$ par $q$ est conjuguée à $\mathrm{T}^{2} \times[0,+\infty[$ et $q$ induit un difféomorphisme de $\tilde{\mathrm{V}}_{2}$ sur son image. Toutes les composantes de bord de $q\left(\tilde{\mathrm{V}}_{1} \cup \mathbf{R} \times \mathrm{S}^{1} \times[-1,1] \cup \tilde{\mathrm{V}}_{2}\right)$ sont des plans et on $a: \overline{\mathrm{V}} \simeq \operatorname{Int}\left(q\left(\tilde{\mathrm{V}}_{1} \cup(\mathbf{R} \times\right.\right.$ $\left.\left.\left.\mathrm{S}^{1} \times[-1,1]\right) \cup \tilde{\mathrm{V}}_{2}\right)\right)$.

- $\quad b_{2}$ ) La composante de Seifert $\mathrm{M}$ adjacente à $\mathrm{T}$ fibre au-dessus d'un ruban de Möebius et deux relevés consécutifs $\overline{\mathrm{T}}$ et $\overline{\mathrm{T}}^{\prime}$ de $\mathrm{T}$ dans $\overline{\mathrm{V}}$ sont des tores. On note $\overline{\mathrm{V}}_{1}$ et $\overline{\mathrm{V}}_{2}$ les deux composantes de $\pi^{-1}(\mathrm{~V} \backslash \mathrm{T})$ attachées à l'extérieur du produit $\mathrm{P}$ relevant $\mathrm{M}$ et délimité dans $\overline{\mathrm{V}}$ par ces tores. Les composantes de bord de $\overline{\mathrm{V}}_{1}$ et $\overline{\mathrm{V}}_{2}$ autres que $\overline{\mathrm{T}}$ et $\overline{\mathrm{T}}^{\prime}$ sont toutes des plans. La variété $\overline{\mathrm{V}}$ est alors conjuguée à $\operatorname{Int}\left(\overline{\mathrm{V}}_{1} \cup \mathrm{P} \cup \overline{\mathrm{V}}_{2}\right)$.

Démonstration. Dire que deux anneaux proprement plongés et $\pi_{1}$-injectés dans $\mathbf{R} \times \mathrm{S}^{1} \times \mathbf{R}$ bordent un produit est un résultat classique pour lequel on renvoie à [Wa].

On est par hypothèses dans le cas où $\mathrm{T}$ est isotope à un des tores de la décomposition de Jaco-Shalen-Johannson et où une composante de $\mathrm{V} \backslash\left(\cup_{0 \leq i \leq n} \mathrm{~T}_{i}\right)$ adjacente à $\mathrm{T}$ est atorö̈dale (et n'est pas un fibré de Seifert).

Soit $\overline{\mathrm{T}}^{\prime}$ un relevé de $\mathrm{T}$ dans $\overline{\mathrm{V}}$, distinct de $\overline{\mathrm{T}}$, qui est un anneau infini (conjugué à $\mathbf{R} \times \mathrm{S}^{1}$ ) ou un tore. On note $\mathrm{K}$ l'adhérence de la composante de $\overline{\mathrm{V}} \backslash\left(\overline{\mathrm{T}} \cup \overline{\mathrm{T}}^{\prime}\right)$ qui rencontre $\overline{\mathrm{T}}$ et $\overline{\mathrm{T}}^{\prime}$. D'après le théorème de Van Kampen, $\pi_{1}(\mathrm{~K})=\pi_{1}(\overline{\mathrm{V}})=\mathbf{Z}^{2}$. Il existe alors dans $\overline{\mathrm{T}}^{\prime}$ une courbe fermée simple homologue à une courbe fermée simple de $\overline{\mathrm{T}}$. Ces deux courbes bordent une surface dans $\mathrm{K}$ que l'on peut toujours rendre incompressible et qui est donc un anneau, car $\pi_{1}(\mathrm{~K})=\mathbf{Z}^{2}$. Comme une composante du découpage adjacente à $\mathrm{T}$ est atoroïdale (et n'est pas un fibré de Seifert), d'après le théorème de l'anneau VIII.10 de [Ja], 
$\overline{\mathrm{T}}$ et $\overline{\mathrm{T}}^{\prime}$ doivent border un même relevé d'une composante du découpage de JacoShalen-Johannson qui est un fibré de Seifert M. En particulier, tous les relevés de $T$ dans $\bar{V}$ qui ne sont pas des plans doivent border l'adhérence d'une même composante de $\pi^{-1}(\mathrm{~V} \backslash \mathrm{T})$.

- Si $\overline{\mathrm{T}}^{\prime}$ est un tore, la composante de Seifert M possède un revêtement de degré deux conjugué à $\mathrm{T}^{2} \times[0,1]$ : il s'agit d'une fibration en cercles sur un ruban de Möebius (cf. [Ja]). Tous les relevés de $\mathrm{T}$ autres que $\overline{\mathrm{T}}$ et $\overline{\mathrm{T}}^{\prime}$ sont, d'après ce qui précède, des plans. La même étude que celle de la configuration a) permet de terminer la démonstration dans ce cas.

- Si $\bar{T}^{\prime}$ est un anneau, par passage au revêtement, on obtient que tous les relevés de $\mathrm{T}$ dans $\tilde{\mathrm{V}}$ qui ne sont pas des plans doivent border l'adhérence d'une même composante de $p^{-1}(\mathrm{~V} \backslash \mathrm{T})$, ce qui suffit à ramener la démonstration, comme dans le cas du lemme précédent, à une utilisation du théorème de Van Kampen et à des raisonnements élémentaires sur les revêtements: par exemple, en recollant des demi-espaces, judicieusement choisis dans le revêtement universel de $\mathrm{V}$, aux composantes de bord de $\tilde{V}_{1} \cup\left(\mathbf{R} \times \mathrm{S}^{1} \times[-2,2]\right) \cup \tilde{V}_{2}$ (qui sont toutes planes d'après ce qui précède), on obtient un revêtement de $\mathrm{V}$ de groupe $\mathbf{Z}$ qui est donc conjugué à $\tilde{V}$.

\section{Construction de la suite d'exemples}

Plus généralement que dans le cas du tore, une surface $S\left(\neq S^{2}, D^{2}\right)$ plongée dans une variété $\mathrm{V}$ de dimension 3 est dite incompressible si son groupe fondamental s'injecte dans celui de $\mathrm{V}$.

Pour construire des structures de contact tendues, on dispose d'un important travail effectué par D. Gabai [Ga] d'une part et par Eliashberg-Gromov [El3, Gr] et Eliashberg-Thurston [E-T] d'autre part.

Un feuilletage est dit tendu s'il possède une transversale fermée qui rencontre toutes ses feuilles. L'existence des feuilletages tendus a été largement étudiée par D. Gabai. On rappelle qu'une surface (orientable) est dite minimale si elle est incompressible et si elle minimise le genre dans sa classe d'homologie. Ici, la surface n'est pas supposée connexe, mais on suppose qu'aucune composante n'est une sphère ou un disque, et le genre désigne alors la somme des genres des composantes connexes.

Théorème 4.1. [Ga] Soient $\mathrm{V}$ une variété compacte irréductible de dimension 3 bordée par une réunion non vide de tores et $\mathrm{S}$ une surface minimale dans $\mathrm{V}$ qui représente un élément non nul de $\mathrm{H}_{2}(\mathrm{~V}, \partial \mathrm{V}, \mathbf{Z})$. Il existe un feuilletage tendu dont une feuille est $\mathrm{S}$ et qui trace sur $\partial \mathrm{V}$ un feuilletage sans singularité ni composante de Reeb.

Un feuilletact est un champ de plans $\xi=\operatorname{ker} \alpha$ tel que la 3 -forme $\alpha \wedge d \alpha$ 
soit de signe constant. Cette notion interpole entre les notions de feuilletage (où $\alpha \wedge d \alpha$ est identiquement nulle) et de structure de contact (où $\alpha \wedge d \alpha$ ne s'annulle pas).

Théorème 4.2. [E-T] Tout feuilletact $\xi$ est limite $\mathrm{C}^{0}$ de structures de contact qui coïncident avec $\xi$ sur un compact où il est déjà de contact.

Pour prouver qu'une variété de contact $(\mathrm{V}, \xi)$ est tendue, on dispose d'un critère dû à Y. Eliashberg et M. Gromov [El3, Gr]: il suffit qu'il existe une variété symplectique $(\mathrm{W}, \tilde{\omega})$ ( $\tilde{\omega}$ est une 2 -forme fermée non dégénérée) qui remplisse $(\mathrm{V}, \xi)$, ce qui signifie que $\partial \mathrm{W}=\mathrm{V}$, que $\left.\tilde{\omega}\right|_{\xi}$ est non dégénérée et que $\tilde{\omega}$ oriente $\mathrm{V}=\partial \mathrm{W}$ comme $\xi$.

En particulier (voir [E-T]), il suffit qu'il existe une 2 -forme fermée $\omega$ sur V qui domine $\xi$, c'est-à-dire telle que $\left.\omega\right|_{\xi}$ soit non dégénérée, ainsi qu'une structure négative $\xi^{\prime}$ également dominée par $\omega$.

Dans ce cas en effet, la 2 -forme $\tilde{\omega}=p^{*} \omega+\epsilon d s \alpha$ obtenue à partir de $\omega$ sur $\mathrm{V} \times[0,1]-s$ est la coordonnée sur $[0,1], \epsilon$ est un réel positif assez petit, $p: \mathrm{V} \times[0,1] \rightarrow \mathrm{V}$ la projection sur $\mathrm{V}$ et $\alpha=0$ est une équation de $\xi$ - détermine un remplissage symplectique de $(\mathrm{V}, \xi) \amalg\left(\mathrm{V}, \xi^{\prime}\right)$ et le théorème de remplissage de $[\mathrm{El} 3, \mathrm{Gr}]$ assure alors que $\xi$ et $\xi^{\prime}$ sont tendues.

Plus généralement, dans le cas où $(\mathrm{V}, \xi)=\partial(\mathrm{W}, \tilde{\omega})$ n'est pas compacte, il faut supposer de plus, outre le fait que $\left.\tilde{\omega}\right|_{\xi}>0$, qu'il existe une structure presque complexe $\mathrm{J}$ sur W qui préserve $\xi$ et telle que $\tilde{\omega}(*, \mathrm{~J} *)$ soit une métrique riemanienne $g$ avec les propriétés suivantes:

- $g$ est complète ;

- le rayon d'injectivité de $g$ est minoré par un réel strictement positif ;

- la courbure sectionnelle de $g$ est majorée.

On dit alors que $(\mathrm{W}, \tilde{\omega})$ à une géométrie finie à l'infini, et, d'après [El3, E-T, Gr], toute variété de contact bordée par une variété symplectique qui possède une géométrie finie à l'infini est tendue.

Un travail de D. Sullivan [Su] fait le lien entre ces résultats de remplissage pour les structures de contact et le monde des feuilletages: imposer l'existence d'une 2 -forme fermée $\omega$ dont la restriction au plan tangent d'un feuilletage est non dégénérée équivaut à dire que ce feuilletage est tendu.

Une autre stratégie pour construire des structures tendues est de déterminer leur comportement par rapport à certaines opérations de chirurgies.

Théorème 4.3. [Co1] Soient $(\mathrm{V}, \xi)$ une variété de contact et $\mathrm{T} \subset \mathrm{V}$ un tore incompressible. Si $\xi \mathrm{T}$ est un feuilletage linéaire et si la variété $\left(\mathrm{V} \backslash \mathrm{T},\left.\xi\right|_{\mathrm{V} \backslash \mathrm{T}}\right)$ est universellement tendue, alors $(\mathrm{V}, \xi)$ l'est aussi. La conclusion est la même si $\mathrm{T}$ est un anneau incompressible plongé qui possède un voisnage tubulaire conjugué à: $\left(\mathbf{R} \times \mathrm{S}^{1} \times[-\epsilon, \epsilon]=\{(x, y, \theta)\}, \operatorname{ker}\left(\cos \left(\theta+\theta_{0}\right)+\sin \left(\theta+\theta_{0}\right)\right)\right)$, avec $\epsilon, \theta_{0} \in \mathbf{R}$.

C'est sur cette chaîne de résultats qu'on se fonde pour prouver le lemme suivant, légère adaptation d'un énoncé de K. Honda, W. Kazez et G. Matić [HKM], qui précise le travail d'Eliashberg et Thurston. 
Lemme 4.4. (voir $[\mathrm{HKM}]$ ) Soit $\mathrm{V}$ une variété orientée de dimension 3, qui est irréductible, compacte et dont le bord est une réunion non vide de tores incompressibles. Soit S une surface minimale plongée dans $\mathrm{V}$, chaque composante connexe de $\mathrm{S}$ étant de bord non vide plongé dans $\partial \mathrm{V}$. Il existe une structure de contact universellement tendue positive $\xi$ sur $\mathrm{V}$, qui trace sur $\partial \mathrm{V}$ un feuilletage linéaire et sur chaque composante de $\mathrm{S}$ un feuilletage transverse au bord avec un signe constant, sans orbite périodique et dont toutes les singularités sont de même signe.

Démonstration. D'après le théorème 4.1 de Gabai, il existe un feuilletage tendu $\mathrm{F}$ dont $\mathrm{S}$ est une feuille et qui trace sur $\partial \mathrm{V}$ un feuilletage sans singularité ni composante de Reeb. Quitte à modifier $\mathrm{F}$, on peut supposer qu'il est conjugué a un feuilletage produit $\mathrm{S} \times[-1,1]$ près de $\mathrm{S} \simeq \mathrm{S} \times\{0\}$.

Soit alors $\omega$ une 2 -forme fermée, donnée par [Su], qui domine $\mathrm{F}$. On modifie $\omega$ près de chaque composante $\mathrm{T}$ de $\partial \mathrm{V}$ de la manière suivante. Soit $\mathrm{T} \times[-1,0]=$ $\{(x, y, \theta)\}$ un système de coordonnées près de $\mathrm{T} \simeq \mathrm{T} \times\{0\}$ tel que $\mathrm{F}$ soit tangent à $\partial_{\theta}$ et transverse à $\partial_{y}(\mathrm{~F} \cap \mathrm{T}$ est sans composante de Reeb). On suppose de plus que $\mathrm{S} \times[-1,1] \cap \mathrm{T}$ est donné par l'équation $d y=0$. La 2 -forme $\omega$ est fermée et s'écrit donc dans ces coordonnées: $\omega=a d x \wedge d y+d \eta$ pour un certain réel $a$ et une certaine 1 -forme $\eta$.

Si $g:[-1,0] \rightarrow \mathbf{R}$ est une fonction positive, nulle près de -1 et strictement positive sur $\left[-\frac{1}{2}, 0\right]$, qui vaut 1 au voisinage de 0 , et si $\chi:[-1,0] \rightarrow \mathbf{R}$ vaut 1 sur $\left[-1,-\frac{1}{4}\right]$ et 0 près de 0 , on pose, pour $t \in \mathbf{R}$,

$$
\omega_{t}=a d x \wedge d y+d(\chi \eta)+t g(\theta) d \theta \wedge d x .
$$

Cette forme $\omega_{t}$ est fermée, coïncide avec $\omega$ près de $\mathrm{T} \times\{-1\}$ et, si $t$ est assez grand, domine $\mathrm{F}$. On fixe un tel réel $t$, assez grand pour chaque composante de bord, et on note $\omega_{t}^{\prime}$ la 2 -forme obtenue en raccordant $\omega_{t}$ à $\omega$ près de chaque composante de bord. On colle alors à chaque composante de bord $\mathrm{T}$ un produit $\mathrm{T} \times[0,+\infty[=\{(x, y, \theta)\} \quad$ (les coordonnées prolongent celles déjà construites sur $\mathrm{T} \times[-1,0])$ sur lequel on prolonge $\mathrm{F}$ par un feuilletage produit et $\omega_{t}^{\prime}$ par la 2 -forme $a d x \wedge d y+t d \theta \wedge d x$. On note $\mathrm{V}^{\prime}$ cette nouvelle variété, $\mathrm{F}^{\prime}$ le nouveau feuilletage et $\omega_{t}^{\prime \prime}$ la nouvelle forme.

On revient à présent sur $\mathrm{V}$. On trace sur $\mathrm{S}$ un feuilletage orienté L transverse à $\partial \mathrm{S}$, dont les singularités sont des selles ou des foyers de divergence positive (pour une certaine orientation de $\mathrm{S}$ fixée) et ne présentant pas d'orbite périodique. On choisit L sortant le long de $\partial \mathrm{S}$. Un tel feuilletage est donné par le noyau d'une 1 -forme $\beta$ avec $d \beta>0$ (voir par exemple [Gi2]). Soit alors $f:[-1,1] \rightarrow \mathbf{R}$ une fonction lisse, nulle en \pm 1 et strictement positive à l'intérieur. Si $t$ désigne la coordonnée transverse à $\mathrm{S}$ dans son voisinage $\mathrm{S} \times[-1,1]$, la 1 -forme $\alpha=$ $d t+f(t) \beta$ vérifie: $\alpha \wedge d \alpha=f(t) d t \wedge d \beta$. Le noyau de $\alpha$ est donc une structure de contact sur $\mathrm{S} \times$ ] $-1,1[$ qui trace $\mathrm{L}$ sur $\mathrm{S}$, et coïncide avec $\mathrm{F}$ au bord. On construit ainsi sur $\mathrm{V}$ un feuilletact positif $\xi_{0}$ qui trace le feuilletage désiré sur $\mathrm{S}$. La proximité de $\xi_{0}$ à $\mathrm{F}$ est donnée par la taille de $f$. 
Soit à nouveau $\mathrm{T}$ une composante de $\partial \mathrm{V}$ et $\left\{x=x_{0}\right\}$ une équation de $\mathrm{S} \cap \mathrm{T}$. Le feuilletage $\xi_{0} \mathrm{~T}$ a une équation de la forme $d y+h(x, y) d x=0$, avec $h\left(x_{0}, y\right)>0$. Soit alors $H: \mathrm{T}^{2} \times[0, \infty[\rightarrow \mathbf{R}$ une fonction lisse qui possède les propriétés suivantes:

- $\frac{\partial H}{\partial \theta}(x, y, \theta)>0$;

- $\lim _{\theta \rightarrow+\infty} H=c_{0}, c_{0} \in \mathbf{R}$;

- $H(x, y, 1)=c_{1}, c_{1} \in \mathbf{R}$;

- $H(x, y, 0)=h(x, y)$.

Le champ de plans défini sur $\mathrm{T}^{2} \times[0,+\infty[$ comme $\{d y+H d x=0\}$ est une structure de contact qui prolonge $\xi_{0}$ en un feuilletact. Elle est transverse à $\partial_{y}$ et trace sur chaque tore $\mathrm{T} \times\{1\}$ un feuilletage linéaire d'équation $d y+c_{1} d x=0$. Elle trace de plus sur $(\mathrm{S} \cap \mathrm{T}) \times[0,1]$ un feuilletage non singulier, dirigé par $\partial_{\theta}$, et donc sans orbite périodique.

On effectue ce prolongement près de chaque composante de $\partial \mathrm{V}$ pour obtenir un feuilletact sur $\mathrm{V}^{\prime}$. Si $t$ est choisi assez grand, la 2 -forme $\omega_{t}^{\prime \prime}$ domine ce champ de plans. Le feuilletact ainsi construit sur $\mathrm{V}^{\prime}$ peut être approximé, d'après le théorème 4.2, par une structure de contact $\xi$. Comme la condition de domination est ouverte pour la topologie $\mathrm{C}^{0}$, la structure $\xi$ est dominée par $\omega_{t}^{\prime \prime}$. Pour construire une structure de contact négative $\xi^{\prime}$ dominée par $\omega_{t}^{\prime \prime}$, il suffit d'appliquer à nouveau le théorème 4.2 pour approximer le feuilletage $\mathrm{F}^{\prime}$ par une structure négative. La variété $V^{\prime}$ n'est pas compacte, mais la forme $\omega_{t}^{\prime \prime}$ est "constante" à l'infini. On peut appliquer le théorème de Gromov et Eliashberg [Gr, El3] pour conclure que $\left(\mathrm{V}^{\prime}, \xi\right)$ est universellement tendue: on s'aperçoit que la forme symplectique $\tilde{\omega}=p^{*} \omega_{t}^{\prime \prime}+\epsilon d s \alpha$ obtenue à partir de $\omega_{t}^{\prime \prime}$ sur $\mathrm{V} \times[0,1]-s$ est la coordonnée sur $[0,1], \epsilon$ est un réel positif assez petit, $p: \mathrm{V} \times[0,1] \rightarrow \mathrm{V}$ la projection sur $\mathrm{V}$ et $\alpha=0$ est une équation de $\xi-$, ainsi que son rappel dans le revêtement universel de $\mathrm{V} \times[0,1]$, a une géométrie finie à l'infini (cf. $[\mathrm{E}-\mathrm{T}]$ ).

Pour conclure, il suffit de remarquer que $\mathrm{V}$ est conjuguée à $\mathrm{V}^{\prime} \backslash\left(\cup_{\mathrm{T} \subset \partial \mathrm{V}} \mathrm{T} \times\right] 1, \infty[)$.

\subsection{Fibrés de Seifert et variétés de type $\mathrm{A}$}

Ici, V désigne une variété irréductible, orientée, close, qui est soit un fibré de Seifert, soit de type A ; $\mathrm{T} \subset \mathrm{V}$ est un tore incompressible défini comme dans la section précédente et $\mathrm{T} \times[-1,1], \mathrm{T} \times\{0\} \simeq \mathrm{T}$, un voisinage tubulaire de $\mathrm{T}$. On note $\mathrm{S} \subset \mathrm{V} \backslash \mathrm{T} \times$ ] - 1, 1[ une surface minimale (dont l'existence est assurée dans $[\mathrm{He}]$ ) qui rencontre les deux tores $\mathrm{T} \times\{ \pm 1\}$ et dont toutes les composantes connexes sont à bord.

D'après le lemme 4.4, il existe une structure de contact universellement tendue $\xi^{0}$ sur $\mathrm{V} \backslash \mathrm{T} \times$ ] $-1,1[$ avec les propriétés suivantes:

- $\xi^{0}$ trace un feuilletage linéaire sur $\mathrm{T} \times\{ \pm 1\}$;

- $\xi^{0}$ est transverse à $\partial \mathrm{S}$ et trace sur $\mathrm{S}$ un feuilletage sans orbite périodique dont toutes les singularités ont le même signe. 
Sur $\mathrm{T} \times[-1,1]$, muni des coordonnées $(x, y, \theta)$, on prolonge $\xi^{0}$ par une structure d'équation $\cos f_{n}(\theta) d x+\sin f_{n}(\theta) d y=0$, où $f_{n}[-1,1] \rightarrow \mathbf{R}$ est une fonction de dérivée strictement positive qui vérifie: $f_{n}(-1) \in\left[0,2 \pi\left[; f_{n}(1) \in[2 n \pi, 2(n+\right.\right.$ 1) $\pi[$, ces valeurs étant telles que les feuilletages tracés sur $T \times\{ \pm 1\}$ par les structures situées de part et d'autre coïncident.

D'après le théorème de recollement 4.3 , toute structure $\xi_{n}$ ainsi construite sur $\mathrm{V}$ est universellement tendue.

\subsection{Variétés de type B}

Ici, chaque composante de $\mathrm{V} \backslash\left(\cup_{0 \leq i \leq n} \mathrm{~T}_{i}\right)$ est un fibré de Seifert et $\mathrm{T}=\mathrm{T}_{0}$. Pour $i=0, \ldots, n$, on note $\mathrm{T}_{i} \times[-1,1], \mathrm{T}_{i} \simeq \mathrm{T}_{i} \times\{0\}$, un voisinage tubulaire de $\mathrm{T}_{i}$ (ces voisinages sont choisis deux à deux disjoints). Toute composante de $\mathrm{V} \backslash$ $\left(\cup_{0 \leq i \leq n} \mathrm{~T}_{i} \times\right]-1,1[)$ est un fibré de Seifert à bord non vide: c'est également un fibré en surfaces (éventuellement tordu) sur le cercle [Ja], toutes les surfaces intersectant transversalement les cercles de la fibration de Seifert. D'après [Co1, Gi2, E-T], il existe une structure de contact universellement tendue $\xi^{0}$ sur $\mathrm{V} \backslash\left(\cup_{0 \leq i \leq n} \mathrm{~T}_{i} \times\right]-$ $1,1[)$, obtenue par déformation du feuilletage donné par les fibres de la fibration sur le cercle, qui trace un feuilletage linéaire sur chaque tore $T_{i} \times\{ \pm 1\}$ et qui est transverse aux fibres de la fibration de Seifert. On complète $\xi^{0}$ sur chaque produit $\mathrm{T}_{i} \times[-1,1], i \neq 0$, par une structure dont l'équation est de la forme (quitte à reparamétrer $\mathrm{T}_{i} \times[-1,1]$ pour que le feuilletage $\xi^{0} \mathrm{~T}_{i} \times\{ \pm 1\}$ soit donné par une équation de ce type): $\cos f^{i}(\theta) d x+\sin f^{i}(\theta) d y=0$.

De la même manière que précédemment, on prolonge la structure ainsi obtenue sur $\mathrm{V} \backslash(\mathrm{T} \times[-1,1])\left(\mathrm{T}=\mathrm{T}_{0}\right)$, par une structure d'équation $\cos f_{n}(\theta) d x+$ $\sin f_{n}(\theta) d y=0$ sur $\mathrm{T} \times[-1,1]$, où $f_{n}:[-1,1] \rightarrow \mathbf{R}$ est une fonction de dérivée strictement positive qui vérifie: $f_{n}(-1) \in\left[0,2 \pi\left[; f_{n}(1) \in[2 n \pi, 2(n+1) \pi[\right.\right.$. On note $\xi_{n}$ cette structure. Elle est universellement tendue d'après le théorème 4.3 .

\subsection{Traduction du théorème 1.1}

Théorème 4.5. La suite $\left(\xi_{n}\right)_{n \in \mathbf{N}}$ comporte une infinité de structures de contact deux à deux non isomorphes.

On note $\mathrm{C}$ la classe d'isotopie du tore $\mathrm{T}$ dans $\mathrm{V}$. Pour montrer le théorème 4.5, il suffit de démontrer le résultat suivant:

Théorème 4.6. $\operatorname{Tor}\left(\mathrm{V}, \xi_{n}, \mathrm{C}\right)<\infty$.

Voici, en effet, comment déduire le théorème 4.5 du théorème 4.6.

D'après [Co2], si $\xi$ est une structure universellement tendue sur V seul un nombre fini (à reparamétrisation près) de classes d'isotopie $\mathrm{D}$ de plongements de $\mathrm{T}^{2} \times[0,2 \pi]$ dans $\mathrm{V}$ fournissent une torsion $\operatorname{Tor}(\mathrm{V}, \xi, \mathrm{D})$ non nulle. On note 
$\left(\mathrm{C}_{i}^{n}\right)_{1 \leq i \leq k_{n}}$ ces classes pour $\left(\mathrm{V}, \xi_{n}\right), \mathrm{C}=\mathrm{C}_{0}^{n}$, et

$$
\mathrm{T}_{n}=\sup _{1 \leq i \leq k_{n}}\left\{\operatorname{Tor}\left(\mathrm{V}, \xi_{n}, \mathrm{C}_{i}^{n}\right), \text { avec } \operatorname{Tor}\left(\mathrm{V}, \xi_{n}, \mathrm{C}_{i}^{n}\right)<\infty\right\}
$$

qui est bien défini et supérieur à $n$ car $n \leq \operatorname{Tor}\left(\mathrm{V}, \xi_{n}, \mathrm{C}_{0}^{n}\right)<\infty$. Ainsi, si $p \in \mathbf{N}$ est choisi assez grand, $\mathrm{T}_{p}>\mathrm{T}_{n}$. Si $u_{n}$ est une suite d'entiers telle que la suite $\mathrm{T}_{u_{n}}$ soit strictement croissante, on est alors assuré que les structures de contact $\xi_{u_{n}}$ sont deux à deux non isomorphes.

Remarque 4.7. Dans [Co3], on décrit une méthode alternative qui permet de munir toute variété orientable, irréductible et toroïdale d'une structure de contact universellement tendue et qui évite tout recours à l'usage d'un remplissage symplectique non compact.

\section{Preuve du théorème 4.6}

\subsection{Fibrés de Seifert}

Ici, V est un fibré de Seifert. Dans cette situation, V possède un revêtement fini $\hat{\mathrm{V}}$ qui est un fibré en cercles. Quitte à considérer un revêtement de degré 2 de $\hat{\mathrm{V}}$, on se ramène au cas où $\hat{\mathrm{V}}$ fibre au-dessus d'une surface orientable $\mathrm{S}$. Tout rappel $\hat{\mathrm{T}}$ de $\mathrm{T}$ dans $\hat{\mathrm{V}}$ est alors incompressible et est, à isotopie près, ou saturé pour cette fibration, ou transverse à la fibration (cf. [He]).

$\mathrm{Si} \mathrm{T}$ est transverse à la fibration, $\hat{\mathrm{V}}$ est un fibré en tores sur le cercle et donc la torsion de $\hat{\xi_{n}}$ est finie dans la classe de $\hat{\mathrm{T}}$ d'après [Gi3, Gi4, Gi5]. C'est donc aussi le cas de la torsion de $\xi_{n}$ dans la classe de $\mathrm{T}$.

$\mathrm{Si} \mathrm{T}$ est saturé, la base $\mathrm{S}$ est de genre strictement positif. Soit $\alpha$ une courbe fermée simple dans $\mathrm{S}$ telle que $\hat{\mathrm{T}}$ soit isotope au rappel de $\alpha$ dans $\hat{\mathrm{V}}$. On note $\beta$ une courbe fermée simple dans $\mathrm{S}$ (dont l'existence est assurée par la minoration du genre de $\mathrm{S}$ ) que l'on ne peut pas disjoindre de $\alpha$ par isotopie et $\hat{\mathrm{T}}^{\prime}$ le rappel de $\beta$ dans $\hat{V}$. Il n'existe alors pas d'isotopie de disjonction de $\hat{\mathrm{T}}$ et $\hat{\mathrm{T}}^{\prime}$, et $\hat{\mathrm{T}}$ est un tore normal. Ainsi, d'après [Co2], la torsion de $\hat{\xi_{n}}$ dans la classe de $\hat{\mathrm{T}}$ est finie ; c'est donc aussi le cas de la torsion de $\xi_{n}$ dans la classe de T.

\subsection{Variétés de type A}

\section{Configuration a)}

Dans cette partie, on va montrer que $\operatorname{Tor}\left(\mathrm{V}, \xi_{n}, \mathrm{C}\right)$ vaut $n-1, n, n+1$ ou $n+2$.

Soient $\mathrm{T} \subset \mathrm{V}$ un tore incompressible dans la configuration a) et $\xi_{n}$ une structure de contact universellement tendue construite selon la méthode proposée plus haut. On note $\overline{\xi_{n}}$ le rappel de $\xi_{n}$ sur $\overline{\mathrm{V}}$ et $\overline{\mathrm{T}} \times[-1,1]$ le relevé de $\mathrm{T} \times[-1,1]$ qui contient $\overline{\mathrm{T}} \simeq \overline{\mathrm{T}} \times\{0\}$. On note de plus $\overline{\mathrm{V}}_{1}^{\prime}$ et $\overline{\mathrm{V}}_{2}^{\prime}$ les deux relevés de 
$\mathrm{V} \backslash \mathrm{T} \times]-1,1[$ dans $\overline{\mathrm{V}}$ adjacents à $\overline{\mathrm{T}} \times[-1,1]$.

Lemme 5.1. $\left(\overline{\mathrm{V}}, \bar{\xi}_{n}\right)$ est contactomorphe $\grave{a}\left(\operatorname{Int}\left(\overline{\mathrm{V}}_{1}{ }^{\prime} \cup(\overline{\mathrm{T}} \times[-1,1]) \cup{\overline{\mathrm{V}_{2}}}^{\prime}\right)\right.$, $\left.\left.\overline{\xi_{n}}\right|_{\operatorname{Int}\left(\overline{\mathrm{V}}_{1}{ }^{\prime} \cup(\overline{\mathrm{T}} \times[-1,1]) \cup \overline{\mathrm{V}}_{2}{ }^{\prime}\right)}\right)$.

Démonstration. On sait par construction que les relevés non compacts de $\mathrm{T} \times\{ \pm 1\}$ dans $\overline{\mathrm{V}}$ ont des voisinages tubulaires disjoints conjugués à $\left(\mathbf{R}^{2} \times[-\alpha, \alpha], \operatorname{ker}(\cos t d x\right.$ $+\sin t(y))$.

On remarque alors à l'aide du lemme 2.9 (et du lemme 3.2) que la structure $\overline{\xi_{n}}$ est conjuguée, sur chaque composante de $\overline{\mathrm{V}} \backslash \operatorname{Int}\left(\overline{\mathrm{V}}_{1}{ }^{\prime} \cup(\overline{\mathrm{T}} \times[-1,1]) \cup \overline{\mathrm{V}}_{2}{ }^{\prime}\right)$, à $\left(\mathbf{R}^{2} \times[0, \infty[, \operatorname{ker}(\cos t d x+\sin t d y))\right.$. Dans $\overline{\mathrm{V}}$, on a donc un plongement de $\left(\mathbf{R}^{2} \times[-\alpha, \infty[, \operatorname{ker}(\cos t d x+\sin t d y))\right.$ près de chaque composante de bord non compacte de $\overline{\mathrm{V}}_{1}{ }^{\prime}$ et $\overline{\mathrm{V}}_{2}{ }^{\prime}$ (celles-ci étant identifiées avec $\mathbf{R}^{2} \times\{0\}$ ). On conclut grâce à un changement de variable explicite bien connu (voir par exemple [Co1], remarque 4.3), ou par une nouvelle application du lemme 2.9 , qui envoie $\left(\mathbf{R}^{2} \times\right.$ $\left[-\alpha, \infty[, \operatorname{ker}(\cos t d x+\sin t d y))\right.$ sur $\left(\mathbf{R}^{2} \times[-\alpha, 0[, \operatorname{ker}(\cos t d x+\sin t d y))\right.$ en laissant invariant un voisinage de $\mathbf{R}^{2} \times\{-\alpha\}$.

Par la suite, $\mathrm{M}$ désigne la variété $\overline{\mathrm{V}}_{1}{ }^{\prime}$ ou $\overline{\mathrm{V}}_{2}{ }^{\prime}$ privée de ses composantes de bord non compactes, et $\zeta$ la structure induite par $\overline{\xi_{n}}$ sur M. On rappelle que $\mathrm{M}$ est difféomorphe à $\mathrm{T}^{2} \times[0,+\infty[$.

Lemme 5.2. La variété $(\mathrm{M}, \zeta)$ est de torsion nulle.

Démonstration. Quitte à changer l'orientation de $\mathrm{S}$, on suppose que toutes les singularités de $\zeta \mathrm{S}$ sont positives. On note $\mathrm{S}^{\prime}$ un rappel de $\mathrm{S}$ dans $\mathrm{M}$, de groupe fondamental $\mathbf{Z}$. La surface $S^{\prime}$ est difféomorphe à un demi-anneau infini $\mathrm{S}^{1} \times\left[0, \infty\left[\right.\right.$. Son bord est inclus dans celui de M. Le feuilletage de $\mathrm{S}^{\prime}$ est formé de singularités positives, et toute feuille de ce feuilletage aboutit en un temps fini à l'une des singularités. Il contient un unique cycle fermé, parallèle au bord.

Par une isotopie $\mathrm{C}^{0}$-petite de $\mathrm{S}^{\prime}$ on fait apparaître, sur chaque liaison entre deux selles de $\zeta S^{\prime}$, un foyer et une selle positives. On note $S^{\prime \prime}$ cette nouvelle surface. Dans $\zeta \mathrm{S}^{\prime \prime}$ on ne peut pas trouver de chemin formé de liaisons contenant 4 selles consécutives.

Pour tout anneau $\mathrm{A} \subset \mathrm{S}^{\prime \prime}, \partial \mathrm{S}^{\prime \prime} \subset \partial \mathrm{A}$, l'ensemble $\omega_{-}$-limite de A par le flot de $\zeta$ A est un graphe de singularités fini $\Gamma$, formé d'arbres venant se greffer sur le cycle fermé. Soit $s$ une selle de ce graphe et $l$ une séparatrice stable de $s$. La séparatrice $l$ provient alors soit d'un foyer, soit d'une selle $s^{\prime}$. À leur tour, les séparatrices stables de $s^{\prime}$ proviennent soit d'un foyer, soit d'une selle dont chaque séparatrice stable provient alors d'un foyer.

Le graphe $\Gamma$ est donc inclus dans un graphe $\Gamma^{\prime}$ fini dont toutes les extrémités sont des foyers et qui contient toutes les séparatrices stables de ses sommets. Le graphe $\Gamma^{\prime}$ possède alors un voisinage tubulaire (qui est un anneau) de bord transverse à $\zeta \mathrm{A}$.

On déduit de ces remarques qu'il existe une exhaustion de $S^{\prime \prime}$ par une suite d'anneaux emboîtés $\left(\mathrm{A}_{i}\right)_{i \in \mathbf{N}}$, telle que: 
- pour tout $i \in \mathbf{N}, \partial \mathrm{S}^{\prime \prime} \subset \partial \mathrm{A}_{i}$;

- pour tout $i \in \mathbf{N}, \partial \mathrm{A}_{i}$ est transverse à $\zeta$ et le feuilletage $\zeta \mathrm{A}_{i}$ est sortant sur les deux bords.

Comme $\mathrm{A}$ est proprement plongé et $\pi_{1}$-injecté dans $\mathrm{M}$, on peut montrer, à l'aide des techniques développées dans [Wa], que le couple ( $\mathrm{M}, \mathrm{A})$ est conjugué à $\left(\mathrm{S}^{1} \times \mathrm{S}^{1} \times\left[0,+\infty\left[,\{*\} \times \mathrm{S}^{1} \times[0,+\infty[)\right.\right.\right.$. En particulier, il existe une exhaustion de $\mathrm{M}$ par une famille de tores épais (conjugués à $\left.\mathrm{T}^{2} \times[0,1]\right)\left(\mathrm{T}_{i}\right)_{i \in \mathbf{N}}$ telle que:

- $\partial \mathrm{M} \subset \partial \mathrm{T}_{i}$;

- $\mathrm{T}_{i}$ rencontre transversalement $\mathrm{S}^{\prime \prime}$ le long de $\mathrm{A}_{i}$.

D'après le lemme 2.6 déduit des travaux de E. Giroux, on peut supposer de plus que $\partial \mathrm{T}_{i}$ présente un feuilletage sans singularité ni composante de Reeb.

Pour conclure, on remarque que chaque anneau $\mathrm{A}_{i}$ porte un arc legendrien $\gamma_{i}$, défini comme réunion de feuilles de $\zeta \mathrm{A}_{i}$, qui joint un bord de $\mathrm{A}_{i}$ à l'autre, et tel que $t b\left(\gamma_{i}, \mathrm{~A}_{i}\right)=-\frac{1}{2}$ car toutes les singularités de $\zeta \mathrm{A}_{i}$ ont le même signe. En particulier, le lemme 2.3 permet de conclure que la torsion de $\mathrm{T}_{i}$ est nulle pour tout $i$. C'est donc aussi le cas pour M.

On applique le lemme 2.2 d'addition des torsions à la décomposition:

$$
\operatorname{Int}\left(\overline{\mathrm{V}}_{1}^{\prime} \cup(\overline{\mathrm{T}} \times[-1,1]) \cup \overline{\mathrm{V}}_{2}^{\prime}\right) .
$$

On note que la torsion de $\left(\overline{\mathrm{T}} \times[-1,1],\left.\overline{\xi_{n}}\right|_{\overline{\mathrm{T}} \times[-1,1]}\right)$ est $n-1$ ou $n$ d'après le lemme 2.1 et que celle des deux autres composantes de la décomposition est nulle d'après le lemme 5.2. On en déduit que la torsion de $\operatorname{Int}\left(\overline{\mathrm{V}}_{1}^{\prime} \cup(\overline{\mathrm{T}} \times[-1,1]) \cup\right.$ $\left.\left.\overline{\mathrm{V}}_{2}{ }^{\prime}\right),\left.\bar{\xi}_{n}\right|_{\mathrm{I} n t\left(\overline{\mathrm{V}}_{1}{ }^{\prime} \cup(\overline{\mathrm{T}} \times[-1,1]) \cup \overline{\mathrm{V}}_{2}{ }^{\prime}\right)}\right)$ vaut $n-1, n, n+1$ ou $n+2$. D'après le lemme 5.1 , c'est aussi le cas pour $\left(\overline{\mathrm{V}}, \overline{\xi_{n}}\right)$.

On conclut en remarquant que:

$$
n-1 \leq \operatorname{Tor}\left(\mathrm{V}, \xi_{n}, \mathrm{C}\right) \leq \operatorname{Tor}\left(\overline{\mathrm{V}}, \overline{\xi_{n}}\right)
$$

\section{Configuration b)}

\section{i. Cas $\left.b_{1}\right)$}

Cette fois, on suppose qu'il existe un revêtement $p: \tilde{V} \rightarrow \mathrm{V}$ de $\mathrm{V}$ conjugué au produit $\mathbf{R} \times \mathrm{S}^{1} \times \mathbf{R}$ où $\mathrm{T}$ possède deux relevés $\tilde{\mathrm{T}}_{1}$ et $\tilde{\mathrm{T}}_{2}$ qui sont des anneaux. Dans la construction de $\xi_{n}$ sur $\mathrm{V}$, on part d'un voisinage tubulaire $\mathrm{T} \times[-1,1]$ de $T$. Dans $\tilde{V}$, on note $\tilde{T}_{1} \times[-1,1]$ et $\tilde{T}_{2} \times[-1,1]$ les deux voisinages de $\tilde{T}_{1}$ et $\tilde{T}_{2}$ qui relèvent $\mathrm{T} \times[-1,1]$. D'après le lemme 3.3 , on peut supposer que $\tilde{\mathrm{V}}=\mathbf{R} \times \mathrm{S}^{1} \times \mathbf{R}$ avec, pour tout $t \in[-1,1], \quad \tilde{\mathrm{T}}_{1} \times\{t\}=\mathbf{R} \times \mathrm{S}^{1} \times\{-2+t\}$ et $\tilde{\mathrm{T}}_{2} \times\{t\}=\mathbf{R} \times \mathrm{S}^{1} \times\{2+t\}$. On note $\tilde{\mathrm{V}}_{1}^{\prime}$ et $\tilde{\mathrm{V}}_{2}^{\prime}$ les deux composantes de $p^{-1}(\mathrm{~V} \backslash(\mathrm{T} \times]-1,1[))$ adjacentes à, respectivement, $\tilde{\mathrm{T}}_{1} \times[-1,1]$ et $\tilde{\mathrm{T}}_{2} \times[-1,1]$ et qui ne rencontrent pas le produit $\left.\mathbf{R} \times \mathrm{S}^{1} \times\right]-3,3[$.

On remarque que pour $i=1,2$, il existe $j_{i} \in\{1,2\}$ tel que $\tilde{\mathrm{V}}_{i}{ }^{\prime}$ revête $\overline{\mathrm{V}}_{j_{i}}{ }^{\prime}$. On note $p_{j_{i}}$ l'application de revêtement. 
Comme, hormis $\tilde{\mathrm{T}}_{i} \times\{ \pm 1\}$, les composantes de $\partial \tilde{\mathrm{V}}_{i}{ }^{\prime}$ sont des plans, la même démonstration que celle du lemme 5.1, basée sur le lemme 2.9 de classification des structures tendues sur le demi-espace, fournit que les sous-variétés $\left(\mathbf{R} \times \mathrm{S}^{1} \times\right]-$ $\left.\infty,-3], \tilde{\xi_{n}}\right)$ et $\left(\mathbf{R} \times \mathrm{S}^{1} \times\left[3,+\infty\left[, \tilde{\xi_{n}}\right)\right.\right.$ de $\tilde{\mathrm{V}}=\mathbf{R} \times \mathrm{S}^{1} \times \mathbf{R}$ sont conjuguées aux variétés $\left(\tilde{V}_{1}{ }^{\prime},\left.\tilde{\xi}_{n}\right|_{\tilde{V}_{1}{ }^{\prime}}\right)$ et $\left(\tilde{V}_{2}{ }^{\prime},\left.\tilde{\xi}_{n}\right|_{\tilde{V}_{2}{ }^{\prime}}\right)$ privées de leurs composantes de bord non annulaires, et donc que:

$$
\left(\tilde{\mathrm{V}}, \tilde{\xi_{n}}\right) \simeq\left(\operatorname{Int}\left(\tilde{\mathrm{V}}_{1}{ }^{\prime} \cup\left(\mathbf{R} \times \mathrm{S}^{1} \times[-3,3]\right) \cup \tilde{\mathrm{V}}_{2}{ }^{\prime}\right),\left.\tilde{\xi}_{n}\right|_{\operatorname{Int}\left(\tilde{\mathrm{V}}_{1}{ }^{\prime} \cup\left(\mathbf{R} \times \mathrm{S}^{1} \times[-3,3]\right) \cup \tilde{\mathrm{V}}_{2}{ }^{\prime}\right)}\right) .
$$

On note $q$, comme dans le lemme 3.3, l'application de revêtement de $\tilde{\mathrm{V}}$ sur $\overline{\mathrm{V}}$ qui envoie $\tilde{\mathrm{T}}_{1}$ sur $\overline{\mathrm{T}}$. De la même façon que précédemment, comme, d'après le lemme 3.3, toutes les composantes de $\partial\left(q\left(\operatorname{Int}\left(\tilde{\mathrm{V}}_{1}{ }^{\prime} \cup\left(\mathbf{R} \times \mathrm{S}^{1} \times[-3,3]\right) \cup \tilde{\mathrm{V}}_{2}{ }^{\prime}\right)\right)\right.$ sont des plans, on obtient que:

$$
\left(\overline{\mathrm{V}}, \overline{\xi_{n}}\right) \simeq\left(q\left(\operatorname{Int}\left(\tilde{\mathrm{V}}_{1}{ }^{\prime} \cup\left(\mathbf{R} \times \mathrm{S}^{1} \times[-3,3]\right) \cup \tilde{\mathrm{V}}_{2}{ }^{\prime}\right)\right),\left.q_{*} \tilde{\xi}_{n}\right|_{\operatorname{Int}\left(\tilde{\mathrm{V}}_{1}{ }^{\prime} \cup\left(\mathbf{R} \times \mathrm{S}^{1} \times[-3,3]\right) \cup \tilde{\mathrm{V}}_{2}\right)} ^{\prime}\right) .
$$

Remarque 5.3. On prendra garde que, si $q\left(\tilde{\mathrm{V}}_{1}{ }^{\prime}\right)=\overline{\mathrm{V}}_{1}{ }^{\prime}$ ou $\overline{\mathrm{V}}_{2}{ }^{\prime}$, on n'a pas en revanche $q\left(\tilde{\mathrm{V}}_{2}{ }^{\prime}\right)=\overline{\mathrm{V}}_{2}^{\prime}$ ou $\overline{\mathrm{V}}_{1}{ }^{\prime}$.

Pour simplifier les notations, on suppose que $q\left(\tilde{V}_{1}{ }^{\prime}\right)=\bar{V}_{1}^{\prime}$, et donc en particulier que $j_{1}=1$.

On veut montrer que la torsion est finie sur $\overline{\mathrm{V}}$. D'après l'isomorphisme précédent, il suffit de considérer un produit $\mathrm{P}=\mathrm{T}^{2} \times[0,2 \pi]$ plongé de manière $\pi_{1}$ injective dans $\left(q\left(\operatorname{Int}\left(\tilde{\mathrm{V}}_{1}{ }^{\prime} \cup\left(\mathbf{R} \times \mathrm{S}^{1} \times[-3,3]\right) \cup \tilde{\mathrm{V}}_{2}{ }^{\prime}\right)\right),\left.q_{*} \tilde{\xi}_{n}\right|_{\operatorname{Int}\left(\tilde{\mathrm{V}}_{1}{ }^{\prime} \cup\left(\mathbf{R} \times \mathrm{S}^{1} \times[-3,3]\right) \cup \tilde{\mathrm{V}}_{2}{ }^{\prime}\right)}\right)$.

Soient $\bar{V}_{1}{ }^{\prime \prime}$ et $\bar{V}_{2}{ }^{\prime \prime}$, les sous-variétés de $\overline{\mathrm{V}}$ obtenues en retirant à $\overline{\mathrm{V}}_{1}^{\prime}$ et $\overline{\mathrm{V}}_{2}{ }^{\prime}$ les composantes de bord autres que $\overline{\mathrm{T}} \times\{ \pm 1\}$. On rappelle que, d'après [Si], pour $i=1,2, \overline{\mathrm{V}}_{i}^{\prime \prime} \simeq \mathrm{T}^{2} \times[0,+\infty[$. D'après l'étude du cas a), et notamment le lemme 5.2, la torsion est nulle sur $\overline{\mathrm{V}}_{i}{ }^{\prime \prime}$.

On remarque alors que si $k_{1}, k_{2} \in \mathbf{N}$ sont assez grand, $\mathrm{P} \cap q\left(\tilde{\mathrm{V}}_{i}^{\prime}\right)$ est inclus dans $\mathrm{T}^{2} \times\left[0, k_{1}\right] \subset \overline{\mathrm{V}}_{1}^{\prime \prime} \simeq \mathrm{T}^{2} \times\left[0,+\infty\left[\right.\right.$ si $i=1$, et dans $p_{j_{2}}^{-1}\left(\mathrm{~T}^{2} \times\left[0, k_{2}\right]\right) \subset$ $q\left(\tilde{\mathrm{V}}_{2}\right) \simeq \tilde{\mathrm{V}}_{2}$ pour $i=2$, où $\mathrm{T}^{2} \times\left[0, k_{2}\right] \subset \overline{\mathrm{V}}_{i_{2}}{ }^{\prime \prime} \simeq \mathrm{T}^{2} \times[0,+\infty[$.

De plus, d'après le lemme 2.4, il existe $\left(c_{1}, c_{2}, \theta_{1}, \theta_{2}\right) \in \mathbf{R}^{4}$ (indépendant de $k_{1}$ et $\left.k_{2}\right)$ tel que, pour $i=1,2,\left(\mathrm{~T}^{2} \times\left[0, k_{i}\right],\left.\overline{\xi_{n}}\right|_{\mathrm{T}^{2} \times\left[0, k_{i}\right]}\right)$ se plonge dans $\left(\mathrm{T}^{2} \times\right.$ $\left.\left[0, c_{i}\right], \operatorname{ker}\left(\cos \left(\theta+\theta_{i}\right) d x+\sin \left(\theta+\theta_{i}\right) d y\right)\right)$.

Ainsi, par passage au revêtement, on en déduit que le rappel par $q$ de $\mathrm{P}$ dans $\tilde{\mathrm{V}}$ se plonge dans le modèle

$$
\begin{gathered}
(\mathrm{W}, \zeta) \simeq\left(\mathbf{R} \times \mathrm{S}^{1} \times\left[0, c_{1}\right], \operatorname{ker}\left(\cos \left(\theta+\theta_{1}\right) d x+\sin \left(\theta+\theta_{1}\right) d y\right)\right) \\
\cup\left(\mathbf{R} \times \mathrm{S}^{1} \times[-3,3],\left.\tilde{\xi_{n}}\right|_{\mathbf{R} \times \mathrm{S}^{1} \times[-3,3]}\right) \\
\cup\left(\mathbf{R} \times \mathrm{S}^{1} \times\left[0, c_{2}\right], \operatorname{ker}\left(\cos \left(\theta+\theta_{2}\right) d x+\sin \left(\theta+\theta_{2}\right) d y\right)\right)
\end{gathered}
$$

(où on identifie $\mathbf{R} \times \mathrm{S}^{1} \times\{0\}$ avec $\mathbf{R} \times \mathrm{S}^{1} \times\{-3\}$ d'une part, et avec $\mathbf{R} \times \mathrm{S}^{1} \times\{3\}$ d'autre part). La variété $(\mathrm{W}, \zeta)$ est obtenue par collage de trois variétés de contact universellement tendues le long d'anneaux $\left(\mathbf{R} \times \mathrm{S}^{1} \times\{ \pm 3\}\right)$ dans un 
voisinage tubulaire desquels la structure a par construction une équation du type $\cos \left(\theta+\theta_{0}\right) d x+\sin \left(\theta+\theta_{0}\right) d y=0$, où $\theta \in\left[-\epsilon^{\prime}, \epsilon^{\prime}\right]$ désigne la coordonnée transverse. En particulier, la version annulaire du théorème de recollement 4.3 fournit que $(\mathrm{W}, \zeta)$ est universellement tendue.

On est de plus bien dans les hypothèses du lemme 2.8: sa conclusion s'applique et la torsion annulaire est finie sur $(\mathrm{W}, \zeta)$.

On conclut la démonstration du théorème 4.6. en remarquant que:

$$
\operatorname{Tor}\left(\mathrm{V}, \xi_{n}, \mathrm{C}\right) \leq \operatorname{Tor}\left(\overline{\mathrm{V}}, \overline{\xi_{n}}\right) \leq \operatorname{Tor}(\mathrm{W}, \zeta) .
$$

\section{ii. Cas $\left.b_{2}\right)$}

L'étude dans ce cas est essentiellement un mélange de celle des configuration $a$ ) et $b_{1}$ ), c'est pourquoi on se contente d'en résumer les étapes. On note $\bar{V}_{1}^{\prime}$ et $\overline{\mathrm{V}}_{2}^{\prime}$ les deux relevés de $\mathrm{V} \backslash(\mathrm{T} \times]-1,1[)$ dans $\overline{\mathrm{V}}$ inclus dans $\overline{\mathrm{V}}_{1}$ et $\overline{\mathrm{V}}_{2}$. On note $\mathrm{P}^{\prime}$ le produit bordé dans $\overline{\mathrm{V}}$ par les composantes toriques de $\partial \overline{\mathrm{V}}_{i}{ }^{\prime}$. Comme les composantes de bord de $\overline{\mathrm{V}}_{1}{ }^{\prime} \cup \mathrm{P}^{\prime} \cup \overline{\mathrm{V}}_{2}{ }^{\prime}$ sont des plans, on obtient, comme dans l'étude du cas $a$ ), un isomorphisme:

$$
\left(\overline{\mathrm{V}}, \overline{\xi_{n}}\right) \simeq\left(\operatorname{Int}\left(\overline{\mathrm{V}}_{1}{ }^{\prime} \cup \mathrm{P}^{\prime} \cup \overline{\mathrm{V}}_{2}{ }^{\prime}\right),\left.\bar{\xi}_{n}\right|_{\operatorname{Int}\left(\overline{\mathrm{V}}_{1}{ }^{\prime} \cup \mathrm{P}^{\prime} \cup \overline{\mathrm{V}}_{2}{ }^{\prime}\right)}\right) .
$$

Pour conclure il faut rappeler que, pour $i=1,2$, la variété obtenue en retirant à $\bar{V}_{i}{ }^{\prime}$ ses composantes de bord non compactes est conjuguée à $T^{2} \times[0,+\infty[$ et que la torsion y est finie (lemme 5.2). La torsion est également finie sur $\mathrm{P}^{\prime}$, par exemple par application du corollaire 2.8 à un revêtement cyclique de $\mathrm{P}^{\prime}$. On applique alors le lemme d'addition des torsions 2.2 à la décomposition ci-dessus pour obtenir que la torsion de $\left(\overline{\mathrm{V}}, \overline{\xi_{n}}\right)$ est finie, et donc aussi celle de $(\mathrm{V}, \xi)$ dans la classe $\mathrm{C}$ de $\mathrm{T}$.

\subsection{Variétés de type $B$}

On note $\mathrm{V}_{1}$ et $\mathrm{V}_{2}$ les composantes de $\mathrm{V} \backslash\left(\cup_{0 \leq i \leq n} \mathrm{~T}_{i} \times\right]-1,1[)$ adjacentes à $\mathrm{T} \times[-1,1]$ (on peut avoir $\mathrm{V}_{1}=\mathrm{V}_{2}$ ). Ce sont des fibrés de Seifert à bord, qui fibrent également sur le cercle (éventuellement de manière tordue). Si la fibre est un anneau, la composante est conjuguée soit à un produit $\mathrm{T}^{2} \times[0,1]$, soit à un fibré en cercles sur une bande de Möebius. Sinon, comme le bord est incompressible, la fibre est une surface de caractéristique strictement négative.

Si $\mathrm{V}_{1}$ (ou $\mathrm{V}_{2}$ ) est conjugué à un produit $\mathrm{T}^{2} \times[0,1]$, par minimalité de la décomposition, c'est aussi le cas de $\mathrm{V}_{2}$ et $\mathrm{V}$ est un fibré en tores sur le cercle.

Si $V_{1}$ et $V_{2}$ sont des fibrés en cercles au-dessus d'un ruban de Möebius, V possède un revêtement de degré 4 qui fibre en tores sur le cercle.

Dans ces deux cas, le théorème 4.6 découle immédiatement de [Gi3, Gi4, Gi5].

On suppose à présent que ni $\mathrm{V}_{1}$ ni $\mathrm{V}_{2}$ ne sont conjugués à un produit ou à un fibré sur un ruban de Möebius. Si $\alpha$ est une courbe fermée simple dans $\mathrm{T}$ qui n'est isotope ni à une fibre de $V_{1}$ ni à une fibre de $V_{2}$ (vus comme fibrés de Seifert), on note: 
- $\tilde{\mathrm{V}}$ un revêtement de $\mathrm{V}$ de groupe $\mathbf{Z}[\alpha]$;

- $\tilde{T} \times[-1,1]$ un relevé de $\mathrm{T} \times[-1,1]$ dans $\tilde{T}$ conjugué à $\mathbf{R} \times \mathrm{S}^{1} \times[-1,1]$;

- $\tilde{V}_{1}$ et $\tilde{V}_{2}$ les relevés de $\mathrm{V}_{1}$ et $\mathrm{V}_{2}$ dans $\tilde{\mathrm{V}}$ adjacents à $\tilde{\mathrm{T}} \times[-1,1]$.

Comme les fibres de $V_{1}$ et $V_{2}$ (vu comme fibrés - ou fibrés tordus - sur le cercle) sont de caractéristique strictement négative, on vérifie que les composantes de

$$
\partial\left(\tilde{V}_{1} \cup \tilde{T} \times[-1,1] \cup \tilde{V}_{2}\right)
$$

sont toutes des plans. On a alors, de la même façon que dans le lemme 5.1:

$$
\left(\tilde{\mathrm{V}}, \tilde{\xi_{n}}\right) \simeq\left(\operatorname{Int}\left(\tilde{\mathrm{V}}_{1} \cup \tilde{\mathrm{T}} \times[-1,1] \cup \tilde{\mathrm{V}}_{2}\right),\left.\tilde{\xi_{n}}\right|_{\operatorname{Int}\left(\tilde{\mathrm{V}_{1}} \cup \tilde{\mathrm{T}} \times[-1,1] \cup \tilde{\mathrm{V}}_{2}\right)}\right) \text {. }
$$

Par construction, la structure $\xi_{n}$ est transverse à la fibration de $\mathrm{V}_{1}$ et $\mathrm{V}_{2}$, et on vérifie alors aisemment (voir par exemple [Co3]) que $\tilde{\xi_{n}}$ a sur $\tilde{\mathrm{V}}_{i} \simeq \mathbf{R} \times$ $\mathrm{S}^{1} \times[-1,1]=\{(x, y, \theta)\}, i=1,2$, une équation de la forme $\cos h_{i}(x, y, \theta) d x+$ $\sin h_{i}(x, y, \theta) d y=0$, où $h_{i}(x, y, \theta)=h_{i}(\theta)$ sur $\mathbf{R} \times \mathrm{S}^{1} \times[-1,-1+\epsilon](\tilde{\mathrm{T}} \times\{ \pm 1\} \simeq$ $\left.\mathbf{R} \times \mathrm{S}^{1} \times\{-1\}\right)$ car le feuilletage $\xi_{n} \mathrm{~T} \times\{ \pm 1\}$ est linéaire, et où $h_{i}$ est à valeur dans $[0,2 \pi]$. A l'aide du lemme 2.8 , on conclut que la torsion annulaire est finie sur $\left(\tilde{\mathrm{V}}, \tilde{\xi_{n}}\right)$. La torsion de $\left(\mathrm{V}, \xi_{n}\right)$ est donc finie dans la classe de $\mathrm{T}$.

Reste le cas où $V_{1}$ est un fibré en cercles sur un ruban de Möebius et où la fibre de $\mathrm{V}_{2}$ est de caractéristique strictement négative. Sous ces hypothèses, $\mathrm{V}_{1}$ possède un revêtement de degré 2 qui est un produit $\mathrm{T}^{2} \times[0,1]$, et donc $\mathrm{V}$ possède un revêtement de degré 2 dans lequel le rappel $V_{1}^{\prime}$ de $V_{1}$ est un produit. Dans ce revêtement $\mathrm{V}^{\prime}$, on considère les deux rappels $\mathrm{T}_{1}^{\prime} \times[-1,1]$ et $\mathrm{T}_{2}^{\prime} \times[-1,1]$ de $\mathrm{T} \times[-1,1]$ adjacents à $\mathrm{V}_{1}^{\prime}$. On constate alors que $\mathrm{P}^{\prime}=$ $\mathrm{T}_{1}^{\prime} \times[-1,1] \cup \mathrm{V}_{1}^{\prime} \cup \mathrm{T}_{2}^{\prime} \times[-1,1]$ est conjugué au produit $\mathrm{T}^{2} \times[-1,1]$. Dans $\mathrm{V}^{\prime}$, on se ramène au cas précédent en considérant les deux rappels de $V_{2}$ adjacents à $\mathrm{P}^{\prime} \quad\left(\mathrm{P}^{\prime}\right.$ joue le rôle de $\left.\mathrm{T} \times[-1,1]\right)$. On en déduit que la torsion de $\left(\mathrm{V}^{\prime}, \xi_{n}^{\prime}\right)$ dans la classe d'isotopie de $\mathrm{P}^{\prime}$ est finie et donc également que la torsion de $\xi_{n}$ dans la classe $\mathrm{C}$ de $\mathrm{T}$ est finie.

\section{Références}

[Co1] V. Colin, Recollement de variétés de contact tendues, Bull. Soc. math. France 127 (1999), 101-127.

[Co2] V. Colin, Sur la torsion des structures de contact tendues, à paraître dans Ann. Scient. Éc. Norm. Sup.

[Co3] V. Colin, Structures de contact tendues sur les variétés toroïdales et approximation de feuilletages sans composante de Reeb, à paraître dans Topology.

[El1] Y. Eliashberg, Classification of over-twisted contact structures on 3-manifolds, Inv. Math. 98 (1989), 623-637.

[El2] Y. Eliashberg, Contact 3 -manifolds, twenty years since J. Martinet's work, Ann. Inst. Fourier 42 (1992), 165-192.

[El3] Y. Eliashberg, Filling by holomorphic discs and its applications, London Math. Soc. Lect. Notes Ser. 151 (1991), 45-67.

[E-T] Y. Eliashberg, W. Thurston, Confoliations, Univ. Lect. Ser. vol. 13 (1998). 
[Ga] D. Gabai, Foliations and the topology of 3-manifolds, J. Diff. Geom. 18 (1983), 445-503.

[Gi1] E. Giroux, Convexité en topologie de contact, Comment. Math. Helvetici 66 (1991), 18-33.

[Gi2] E. Giroux, Topologie de contact en dimension 3, Sém. Bourbaki 760 (1992-93), 1-27.

[Gi3] E. Giroux, Une structure de contact, même tendue est plus ou moins tordue, Ann. Scient. Éc. Norm. Sup. 27 (1994), 697-705.

[Gi4] E. Giroux, Une infinité de structures de contact tendues sur une infinité de variétés, Invent. Math. 135 (1999), 789-802.

[Gi5] E. Giroux, Structures de contact en dimension 3 et bifurcations des feuilletages de surfaces, Invent. Math. 141 (2000), 615-689.

[Gi6] E. Giroux, Tomographie des structures de contact en dimension 3 , Habilitation de l'Éns Lyon (2000), 1-41.

[Gr] M. Gromov, Pseudo-holomorphic curves in symplectic manifolds, Invent. Math. 82 (1985), 307-347.

[He] J. Hempel, Three manifolds, Ann. Math. Stu. Princeton Univ. Press.

[Ho] K. Honda, On the classification of tight contact structures I: lens spaces, solid tori, and $\mathrm{T}^{2} \times \mathrm{I}$, prépublication (1999).

[HKM] K. Honda, W. Kazez, G. Matić, Tight contact structures and taut foliations, prépublication (2000)

[Ja] W. Jaco, Lectures on three manifolds topology, A.M.S.

[J-S] W. Jaco, P. B. Shalen, Seifert fibered spaces in 3 -manifolds, Mem. AMS 220 (1979).

[Jo] K. Johannson, Homotopy equivalences of 3 -manifolds with boundary, Springer LNM, 761 (1979).

[Ka] Y. Kanda, The classification of tight contact structures on the 3 -torus, Comm. in Anal. and Geom. 5 (1997), 413-438.

[Si] J. Simon, Compactification of covering spaces of compact 3 -manifolds, Michigan Math. J. 23 (1976), 245-256.

[Su] D. Sullivan, A homological characterization of foliations consisting of minimal surfaces, Comment. Math. Helv. 54 (1979), 218-223.

[Wa] F. Waldhausen, On irreducible 3 -manifolds which are sufficiently large, Ann. of Math. 87 (1968), 56-88.

Vincent Colin

Université de Nantes

Département de mathématiques

UMR 6629 du CNRS

2 , rue de la Houssinière

BP 92208

F-44322 Nantes cedex 3

e-mail: Vincent.Colin@math.univ-nantes.fr

(Received: Octobre 16, 2000) 